\title{
An Interactive System for the Visualization and Measurement Extraction in Medical Images
}

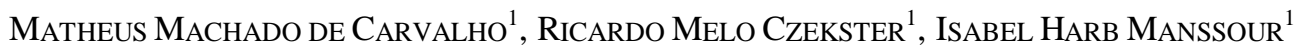 \\ ${ }^{1}$ PUCRS - Pontifícia Universidade Católica do Rio Grande do Sul, Faculdade de Informática \\ Av. Ipiranga 6681, Prédio 30, Bloco 4, 90619-900 Porto Alegre, RS, Brasil \\ \{matheus, ricardo\}@zionsolutions.com.br, manssour@inf.pucrs.br
}

\begin{abstract}
The visualization of medical images, e.g. Computed Tomography (CT) and Magnetic Resonance Imaging (MRI), and the utilization of measurement tools to define distances, areas and volumes are very important to health professionals. The quantitative analysis of spatial relations among structures is useful for medical diagnosis. This work describes a visualization system that provides interactive measurement tools. MRI images acquired from a phantom were used to increase system accuracy.
\end{abstract}

\section{Introduction}

Since the goal of the physicians usually resides in visualizing and quantifying isolated structures inside volume data, it is necessary to provide interactive tools for volume visualization and measurement of structures. Thus, the main goal of this work is to present an interactive visualization system that provides tools for 2$\mathrm{D}$ distance, perimeter and area calculation.

The system is being implemented for Microsoft Windows environment using VTK [1] and Borland C++ Builder to build the interface. It allows the conversion of DICOM data sets to RAW image data, which is the file format supported. For system validation, we used a phantom, a cylindrical device composed by synthetic material and used to test and calibrate medical scanners.

\section{System Architecture and Measurement Tools}

System architecture is based on the MVC pattern [2], which is extensively used in interactive systems to separate the functional core from the user interface. Thus, with a new interface the system could be easily adapted to run, for example, in Linux environment.

The 3-D visualization is being done through the ray casting algorithm provided by VTK. The available measurement tools include distance between two points, region-of-interest (ROI) selection using polilines for area calculation, and ROI perimeter. All these tools are associated with a specific slice, as illustrated in figure 1.

\section{Final Comments and Future Work}

The developed system provides an interactive insertion of ROI, allowing real-time perimeter, distance and area calculation. Measurement results were validate with the phantom data, as shown in Figure 2, where the processed diameter is exactly the same of the real device.

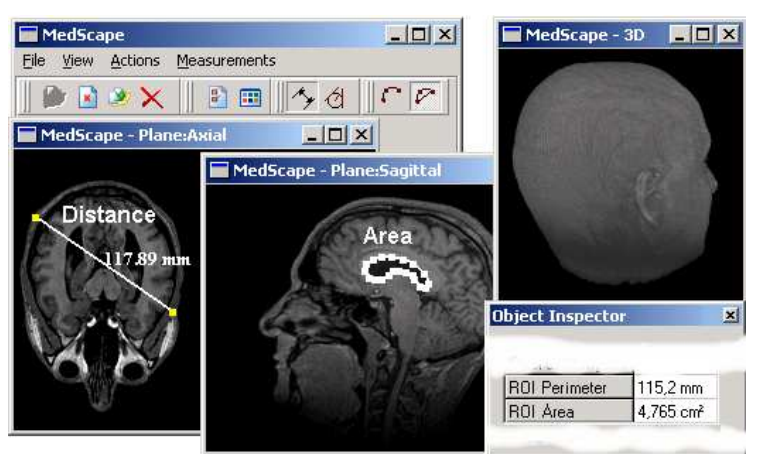

Figure 1 System interface.

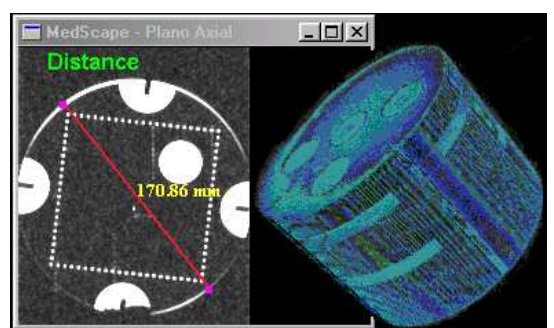

Figure 2 Processed phantom diameter.

Future works include the extension of the measurement tools to allow 3-D measurement. This will invoke the association of a 3-D object with the visualization algorithm to identify the region being analyzed and to allow the calculation of a ROI volume.

\section{References}

[1] W. Schroeder, K. Martin, B. Lorensen. The Visualization Toolkit: An Object-Oriented Approach to 3D Graphics. Prentice Hall, 1998.

[2] F. Buschmann, R. Meunier, H. Rohnert et al. PatternOriented Software Architecture - A System of Patterns, John Wiley \& Sons, 1996. 